\title{
Influence of ultrasound contrast agents on spectral Doppler analysis in recipients of liver transplantation
}

\author{
Young Seo Cho', Kyoung Won Kim ${ }^{2}$, Hye Young Jang ${ }^{2,3}$, Bo Hyun $\mathrm{Kim}^{4}$, Jeongjin Lee ${ }^{5}$, Gi Won Song ${ }^{6}$, Sung Gyu Lee ${ }^{6}$, \\ and Dagvasumberel Munkhbaatar ${ }^{7}$ \\ 'Department of Radiology, Hanyang University Guri Hospital, Guri; ${ }^{2}$ Department of Radiology, Asan Medical Center, University of Ulsan College of \\ Medicine, Seoul; ${ }^{3}$ Department of Radiology, Konyang University Hospital, Konyang University College of Medicine, Daejeon (current affiliation); \\ ${ }^{4}$ Department of Radiology, Ajou University Hospital, Suwon; ${ }^{5}$ School of Computer Science \& Engineering, Soongsil University; ${ }^{6}$ Division \\ of Hepatobiliary and Liver Transplantation Surgery, Department of Surgery, Asan Medical Center, University of Ulsan College of Medicine, \\ Seoul; 'Department of Radiology, School of Medicine, Mongolian National University of Medical Sciences, Ulaanbaatar, Mongolia
}

Background/Aims: Clinical validation is required to determine whether Doppler measurements are comparable before and after administering ultrasound contrast agent (USCA). The purpose of this study is to explore whether the use of USCA affects spectral Doppler analysis in recipients of liver transplantation (LT).

Methods: For this study, 36 patients were examined using Doppler ultrasonography (US) along with a contrastenhanced US for surveillance of vascular complications after LT. The following spectral Doppler US parameters were measured before and after administration of USCA: peak systolic velocity, end-diastolic velocity, resistive index, and systolic acceleration time of the graft hepatic artery; peak flow velocity of the graft portal vein; and peak flow velocity and venous pulsatility index of the graft hepatic vein.

Results: The mean peak systolic and end-diastolic velocities of the hepatic artery and the peak flow velocity of the portal and hepatic veins were increased after intravenously administration of the USCA, ranging from $10 \%$ to $13 \%$. However, the changes were not statistically significant $(P=0.097,0.103,0.128$, and 0.190 , respectively). There were no significant differences in other measured parameters, including the resistive index $(P=0.205)$ and systolic acceleration time $(P=0.489)$ of the hepatic artery and venous pulsatility index $(P=0.494)$ of the hepatic vein.

Conclusions: The measured velocities of graft hepatic vessels tended to increase after administration of USCA, but without statistical significance. The comparison of serial Doppler parameters with or without injection of USCA is valid during Doppler surveillance in recipients of LT. (Clin Mol Hepatol 2017;23:224-229)

Keywords: Doppler shift; Liver transplantation; Microbubbles

\section{INTRODUCTION}

Vascular obstruction is a major complication after liver transplantation (LT). Hepatic artery stenosis is the most threatening complication because it can lead to loss of the grafted liver (3$8 \%)^{.}$Clinical features in recipients with portal and hepatic vein obstructions vary according to the severity of the obstruction. Graft failure is an extreme event reserved for severe cases; and

\section{Abbreviations:}

CEUS, contrast enhanced ultrasound; CT, computed tomography; LT, liver transplantation; USCA, ultrasound contrast agent

\section{Corresponding author : Kyoung Won Kim}

Department of Radiology, Asan Medical Center, University of Ulsan College of Medicine, 88 Olympic-ro 43-gil, Songpa-gu, Seoul 05505, Korea

Tel: +82-2-3010-4385, Fax: +82-2-476-4719

E-mail: kimkw@amc.seoul.kr 
Young Seo Cho, et al.

Doppler parameters in LT after US contrast agent

more commonly, vascular obstruction deteriorates postoperative course, by causing elevation of liver enzymes, ascites, abdominal distention, and gastrointestinal bleeding.

Ultrasonography is the initially accessible imaging technique for the evaluation of vascular complications in the early postoperative period and for long-term follow-up..$^{2-4}$ Doppler sonography is a quick, noninvasive, portable method for assessing the vasculature of a transplanted liver, and the examination can be performed at the patient bedside. Several Doppler parameters are feasible for detecting vascular complications. Recently, the use of contrastenhanced ultrasound (CEUS) has been increased to evaluate the LT patients. The use of ultrasound contrast agent (USCA) makes grayscale contrast enhancement image similar to that of contrastenhanced computed tomography (CT) which enables detection of parenchymal perfusion defects, ischemic changes and vascular congestion. Moreover, it boosts Doppler signals and may aid in evaluating the Doppler spectrum in hepatic vasculatures having weak or otherwise undetectable Doppler signals. ${ }^{5}$

The signals from conventional Doppler examination are reflected from red blood cells. ${ }^{6}$ After administering USCA, the signals are enhanced by the microbubbles. The serial follow-up of Doppler parameters in LT patients is important for the diagnosis of vascular complications (such as hepatic artery steal syndrome and portal steal syndrome) and acute cellular rejection. If Doppler parameters are affected by the administration of USCA on serial follow up, the occurrence and deterioration of liver pathology can be masked in postoperative Doppler examination for LT patients using USCA. Clinical validation is required to determine whether Doppler measurements are comparable before and after administering USCA. This study aimed to compare the spectral Doppler velocities and parameters that were measured before and after administering USCA in an immediate postoperative period for recipients of LT.

\section{MATERIALS AND METHODS}

\section{Subjects}

The study was approved by our institutional review board. They agreed to waive informed consent from each the patients for this retrospective study.

A total of 148 patients underwent $\mathrm{LT}$ at our institution between January 2009 and April 2009 and were referred to the radiology department for routine postoperative Doppler and CEUS evaluation on the first day after transplantation to check for immediate postoperative vascular complications. Patients who were able to initiate blood flow velocity measurement within 5 minutes of administration of the USCA were included. Patients who did not measure all velocity and Doppler parameters of hepatic artery, portal vein, and hepatic vein before and after USCA administration were excluded. Of them, 36 patients (mean age, 48.8 $8 \pm 10.1$ years; range, 15-65 years) from whom Doppler spectrograms were obtained before and after intravenous injection of USCA were included. There were 28 males (mean age, 48.8 10.2 ; range, 15-64 years) and eight females (mean age, 48.8 10.7 ; range, 30-65 years). Of 36 patients, eight underwent deceaseddonor $\mathrm{LT}$, while the remaining underwent living-donor $\mathrm{LT}$ using a modified right lobe $(n=23)$, left lobe $(n=1)$, or dual $(n=4)$ grafts. Indications for $\mathrm{LT}$ included liver cirrhosis associated with hepatitis $B$ or $C$ virus $(n=12)$, hepatocellular carcinoma $(n=15)$, acute fulminant hepatitis $(n=3)$, primary biliary cirrhosis $(n=1)$, chronic rejection after previous transplantation $(n=2)$, Wilson's disease $(n=1)$, and alcoholic liver cirrhosis $(n=2)$.

\section{Doppler and CEUS technique}

All Doppler and CEUS examinations were performed using an Acuson Sequoia $^{\mathrm{TM}} 512$ machine (Acuson Siemens, Mountain View, CA, USA) with a 1-4-MHz convex transducer. For US examination, patients were placed in the supine position with their heads elevated and right arms abducted. Most examinations were performed by oblique intercostal scanning.

Before administration of USCA, spectral Doppler parameters were evaluated as follows. The peak systolic velocity, end-diastolic velocity, resistive index, and systolic acceleration time were measured at the post-anastomotic graft hepatic artery. The peak flow velocity of the graft portal vein and the peak flow velocity and venous pulsatility index of the graft hepatic vein were measured at an available and reproducible region. All measurements were obtained on waveforms that could be reproduced for at least three consecutive heartbeats. For spectral Doppler sampling, the transmit frequency was $3 \mathrm{MHz}$ and the width of sampling volume was $4 \mathrm{~mm}$-fixed. We set the beam-flow angle between $45^{\circ}$ and $60^{\circ}$ if possible for measurement of Doppler velocity.

For CEUS, SonoVue ${ }^{\mathrm{TM}}$ (Bracco Imaging, Milan, Italy) was used as USCA, the second generation USCA which is licensed clinical indication for liver. SonoVue ${ }^{\mathrm{TM}}$ consists of Sulphur hexafluoride encased by a phospholipid, and once prepared, the bubbles remain stable in the vial for several hours. After manually mixing a freeze dried powder bottle of SonoVue ${ }^{T M}$ with $5 \mathrm{~mL}$ of saline, the mixture 
was administered by a bolus injection through the central venous route, followed by flushing with $10 \mathrm{~mL}$ of saline.

After intravenous USCA injection, low mechanical index grayscale harmonic imaging was performed to evaluate vascular structures and parenchymal perfusion. Spectral Doppler measurement was then followed to evaluate the same region of the graft vasculatures that had been measured before contrast, with as close to the same beam-flow angle as possible. The time from intravenous USCA administration to the start of spectral Doppler parameter measurements was recorded. The gain settings were adequately adjusted for spectral Doppler measurement before and after administration of USCA.

\section{Statistical analysis}

The velocities and indices that were obtained using spectral Doppler imaging of the graft hepatic vasculature (peak systolic velocity, end-diastolic velocity, resistive index, and systolic acceleration time of the graft hepatic artery; peak flow velocity of the graft portal vein; and peak flow velocity and venous pulsatility index of the graft hepatic vein) before and after intravenous administration of USCA were expressed as the mean value \pm standard deviation (SD) with difference assessed by the paired $t$-test. The significance level was set at $P<0.05$.

\section{RESULTS}

A summary of the results is presented in Table 1. Before administration of USCA, the mean peak systolic and end-diastolic veloc- ities of the graft hepatic artery were $79.0 \mathrm{~cm} / \mathrm{s}\left(\mathrm{SD}_{1} \pm 45.2 \mathrm{~cm} / \mathrm{s}\right.$; range, $20.1-222.0 \mathrm{~cm} / \mathrm{s})$ and $23.0 \mathrm{~cm} / \mathrm{s}(S D, \pm 19.5 \mathrm{~cm} / \mathrm{s}$; range, $2.5-109.0 \mathrm{~cm} / \mathrm{s}$ ), respectively. The mean systolic acceleration time and resistive index were $65.3 \mathrm{~ms}$ (SD, $\pm 14.4 \mathrm{~ms}$; range, 33-100 $\mathrm{ms})$ and 0.71 (SD, \pm 0.12 ; range, $0.47-1.01$ ), respectively. The mean peak flow velocity of the graft portal vein was $74.3 \mathrm{~cm} / \mathrm{s}$ $(S D, \pm 35.4 \mathrm{~cm} / \mathrm{s}$; range, $13.9-193.0 \mathrm{~cm} / \mathrm{s})$. The mean peak flow velocity and venous pulsatility index of the graft hepatic vein were $47.0 \mathrm{~cm} / \mathrm{s}(S D, \pm 22.6 \mathrm{~cm} / \mathrm{s}$; range, $13.7-118.0 \mathrm{~cm} / \mathrm{s})$ and 0.65 (SD, \pm 0.41 ; range, $0.1-1.7)$, respectively.

After USCA injection, 114-287 s (187.6 \pm 37.1 s) were spent evaluating vascular structures and parenchymal perfusion using low mechanical index grayscale harmonic imaging. Spectral Doppler measurement was thereafter initiated.

After administration of USCA, both peak systolic and end-diastolic velocities increased in $22(61 \%)$ patients and decreased in $14(39 \%)$ patients compared with the velocities obtained before USCA injection. The mean peak systolic and mean end-diastolic velocities of the graft hepatic artery after administering USCA were $89.0 \mathrm{~cm} / \mathrm{s}(S D, \pm 56.0 \mathrm{~cm} / \mathrm{s}$; range, $22.9-312.0 \mathrm{~cm} / \mathrm{s})$ and $25.2 \mathrm{~cm} / \mathrm{s}(S D, \pm 22.1 \mathrm{~cm} / \mathrm{s} ;$ range, $3.3-124.0 \mathrm{~cm} / \mathrm{s})$, respectively. Both values increased on an average by $13 \%$ and $10 \%$, respectively, compared with values that were obtained before USCA injection,; however, the increments were not statistically significant ( $P=0.097$ and 0.103 , respectively) (Fig. 1A).

Both the systolic acceleration time and resistive index of the graft hepatic artery increased in $23(64 \%)$ patients and decreased in $13(36 \%)$ after administering USCA. The mean systolic acceleration time and resistive index after administration of USCA were $67.6 \mathrm{~ms}$ (SD, $\pm 19.7 \mathrm{~ms}$; range, 42-116 ms) and 0.72 (SD, \pm 0.11 ;

Table 1. Differences in mean Doppler values of graft hepatic vasculature before and after administration of USCA

\begin{tabular}{lccc}
\hline & Before USCA & After USCA & P-value \\
\hline HA & & & 0.097 \\
\hline Peak systolic velocity (cm/s) & $79.0 \pm 45.2$ & $89.0 \pm 56.0$ & 0.103 \\
\hline End-diastolic velocity (cm/s) & $23.0 \pm 19.5$ & $25.2 \pm 22.1$ & 0.205 \\
\hline Resistive index & $0.71 \pm 0.12$ & $0.72 \pm 0.11$ & 0.489 \\
\hline Systolic acceleration time (ms) & $65.3 \pm 14.4$ & $67.6 \pm 19.7$ & 0.128 \\
\hline PV & & & $82.0 \pm 39.3$ \\
\hline Peak flow velocity (cm/s) & $74.3 \pm 35.4$ & & 0.190 \\
\hline HV & & $53.3 \pm 27.8$ & 0.494 \\
\hline Peak flow velocity (cm/s) & $47.0 \pm 22.6$ & $0.68 \pm 0.41$ & \\
\hline
\end{tabular}

Values are presented as mean \pm SD unless otherwise indicated.

USCA, ultrasound contrast agent; HA, hepatic artery; PV, portal vein; HV, hepatic vein. 
Young Seo Cho, et al. Doppler parameters in LT after US contrast agent
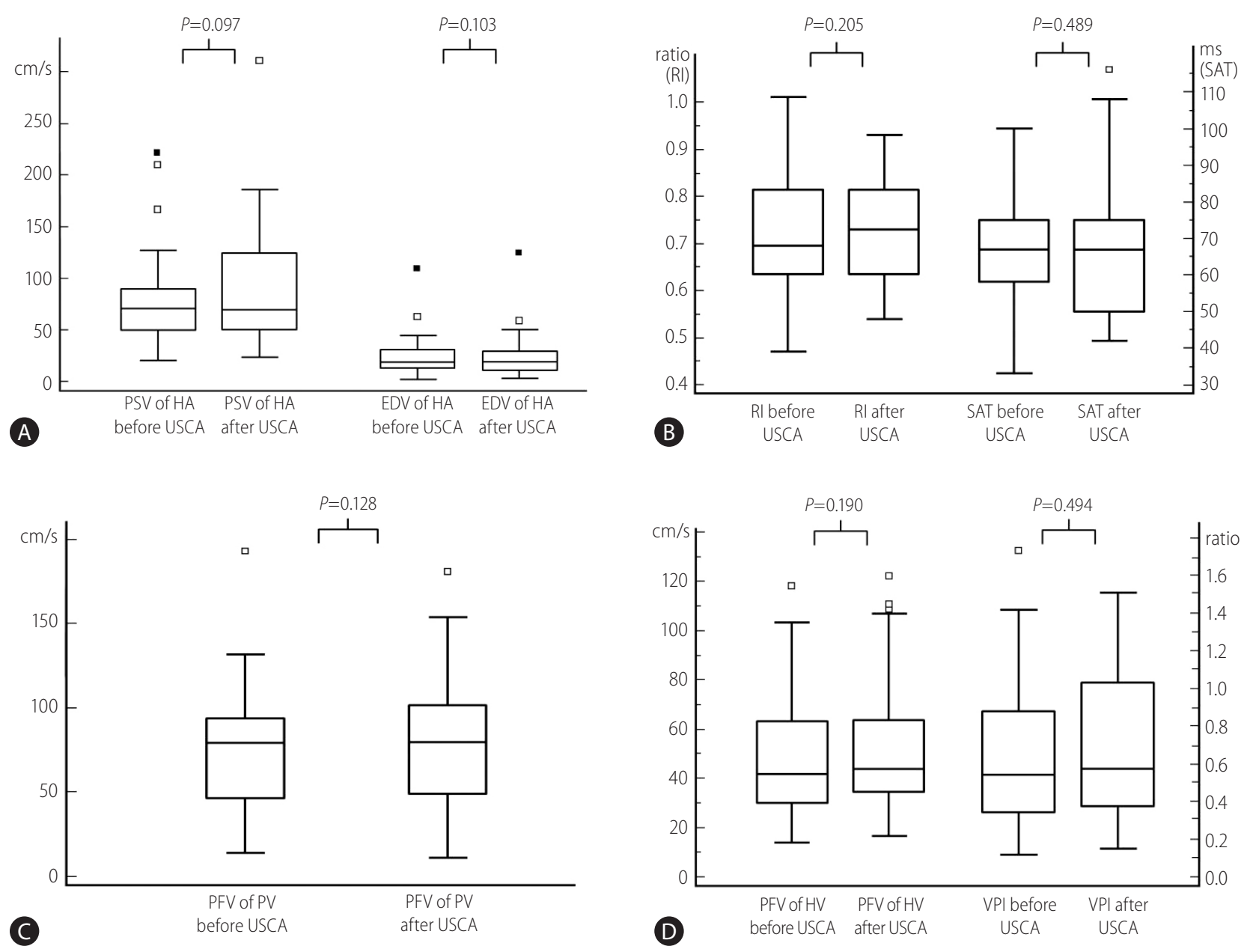

Figure 1. Box-and-whisker plot of the Doppler values of graft hepatic vasculature before and after administration of USCA. (A) The peak systolic velocity and end-diastolic velocity of the hepatic artery before and after administration of USCA. (B) The resistive indices and systolic acceleration time of the hepatic artery before and after administration of USCA. (C) The peak flow velocity of the portal vein before and after administration of USCA. (D) The peak flow velocity and venous pulsatility indices of the hepatic vein before and after administration of USCA. USCA, ultrasound contrast agent; PSV, peak systolic velocity; HA, hepatic artery; EDV, end-diastolic velocity; RI, resistive index; SAT, systolic acceleration time; PFV, peak flow velocity; PV, portal vein; $\mathrm{HV}$, hepatic vein; $\mathrm{VPI}$, venous pulatility index.

range, $0.54-0.93)$. The systolic acceleration time increased on an average by $4 \%$, and the resistive index increased by $1 \%$ after administering USCA, but neither were statistically significant differences ( $P=0.489$ and 0.205 , respectively) (Fig. 1B).

The peak flow velocity of the graft portal vein increased in 22 (61\%) patients and decreased in $14(39 \%)$ after administering USCA. The mean peak flow velocity after administering USCA was $82.0 \mathrm{~cm} / \mathrm{s}(S D, \pm 39.3 \mathrm{~cm} / \mathrm{s}$; range, 10.7-181.0 cm/s) showing $10 \%$ increase compared with that obtained before USCA injection. The difference was not statistically significant $(P=0.128)$ (Fig. $1 C)$. The peak flow velocity of the graft hepatic vein increased in $25(69 \%)$ patients and decreased in $11(31 \%)$ after administering USCA. The mean peak flow velocity of the graft hepatic vein after administering USCA was $53.3 \mathrm{~cm} / \mathrm{s}(S D, \pm 27.8 \mathrm{~cm} / \mathrm{s}$ : range, $16.4-$ $122.0 \mathrm{~cm} / \mathrm{s}$ ), which is a $13 \%$ increase compared with that obtained before USCA injection. The difference was not statistically significant $(P=0.190)$ (Fig. 1D).

The venous pulsatility index of the graft hepatic vein increased in $19(53 \%)$ patients and decreased in $17(47 \%)$ after administration of USCA. The mean venous pulsatility index after administering USCA was 0.68 (SD, \pm 0.41 ; range, $0.13-1.48$ ), showing $5 \%$ increase compared with the value before administering USCA. The difference was not statistically significant $(P=0.494)$ (Fig. 1D). 


\section{DISCUSSION}

We found that although there was a tendency for Doppler velocities in the hepatic artery and portal and hepatic veins to increase after administering USCA, with mean increases of $10-$ $13 \%$, they did not significantly differ from the values that were obtained before USCA. And the calculated Doppler parameters, the resistive index of the graft hepatic artery and venous pulsatility index of graft hepatic vein, were not significantly affected by administering USCA (1\% and 5\% increments, respectively) nor was the graft hepatic artery systolic acceleration time significantly affected by administering USCA (4\% increment).

Whether Doppler velocities change after administering USCA is a controversial topic. According to Forsberg, et al. , after administration of USCA, Doppler velocities increased from 20\% to $45 \%$ in a rabbit aorta model and $17 \%$ in a flow phantom. They supposed that the observed increments in peak velocity measurements represented an artifact that was produced by contrast agents, and this phenomenon was probably related to a strong enhancement of the signal intensity exceeding the dynamic range of their scanner. In a cardiac model reported by Yokoyama, et al. ${ }^{8}$, the Doppler velocity increased by $13 \%$ after administration of USCA. In contrast, other experimental and clinical studies have demonstrated that Doppler velocities were not significantly changed after administering USCA with adequate adjustment of the Doppler gain. ${ }^{9,10}$ Most of the previous studies were conducted using phantom or animal models or evaluated large superficial vessels or intracardiac flow in humans. In this study, we attempted to determine whether measuring Doppler velocities and other parameters is significantly affected by the use of USCA in common clinical settings of postoperative evaluation of recipients of $L T$.

In the current study, the ranges of the Doppler velocity increments that we documented were wider (-61 270\%) than those documented in previous studies. In addition, we frequently encountered a decrease in the Doppler velocities in some patients after administration of USCA, unlike in previous studies. We assumed that the wide range of change in velocity and the finding of velocity decrease in some patients after the use of USCA might have been caused by several confounding factors other than USCA affecting the spectral Doppler velocity measurement. In the clinical setting of performing hepatic Doppler after LT, it may sometimes be difficult to assess the exact direction of blood flow. This difficulty can be encountered, particularly in the case of a small tortuous hepatic artery that may not be traceable on a grayscale image and only discontinuously appears on color flow imaging. Furthermore, if there is a helical or turbulent flow, which is common in the graft portal vein when there is a donor-recipient size discrepancy or mild anastomotic stenosis, the blood flow may not be parallel to the longitudinal axis of the vessels. Also, although Doppler velocity measurements before and after the administration of USCA were performed by the same radiologist in our study, there may have been minute differences in the adequacy of Doppler angle setting between the two examinations. Lastly, exaggerated respiratory motion may have disturbed the stability of Doppler velocity measurement.

In our study, we did not perform the Doppler examination immediately after USCA administration. There was a time lag of 114-287 s, depending on the time spent for the harmonic grayscale enhancement imaging to evaluate the graft vessels and parenchyma. Therefore, a variable degree of metabolic and mechanical breakage of the contrast microbubbles may have occurred before we initiated Doppler measurements, which may have changed the USCA concentration to unknown degree. However, this is unlikely to have caused serious variation in the USCA concentration because the period beginning from $90 \mathrm{~s}$ after administering the contrast is hemodynamically classified as an equilibrium phase following a single bolus intravenous injection of the contrast agent. ${ }^{11}$ And, the low mechanical index used for grayscale harmonic imaging would be unlikely to cause much destruction of microbubbles. Nevertheless, the effects of time interval after USCA administration to the changes of USCA concentration and the Doppler frequency shift are not evaluated before, and a further study with strictly controlled scan time after administration of USCA may be meaningful.

There are some limitations in our study. The first is the variable time elapse before post-contrast Doppler velocity measurement as described above. The second is that we did not evaluate inter-observer variability. Doppler assessment is a repeatable method for measuring hepatic blood flow, with stable intra-observer variability. ${ }^{12}$ However, inter-observer variability of Doppler measurements has been reported to range from $0 \%$ to $10 \% .^{13,14}$ In the usual clinical setting, postoperative serial follow-up Doppler examinations of recipients of $L T$ are not performed by one examiner, and therefore, Doppler parameters before and after administering USCA could be affected by inter-observer variability. However, all Doppler examinations in our study were performed by one examiner, and the inter-ob- 
Young Seo Cho, et al.

Doppler parameters in LT after US contrast agent

server variability was not considered.

In conclusion, the measured velocities of graft hepatic vessels tended to increase after administration of USCA in recipients of $\mathrm{LT}$; however, the differences were not statistically significant. The calculated Doppler parameters (resistive index of hepatic artery and venous pulsatility index of hepatic vein) and the systolic acceleration time of the graft hepatic artery were not significantly affected by administration of USCA. Therefore, the comparison of these parameters between the exams performed with or without administering USCA is valid in Doppler surveillance of recipients of LT.

\section{Author contribution}

Study conception and design: Kyoung Won Kim

Acquisition of data: Kyoung Won Kim, Young Seo Cho, Hye Young Jang

Analysis and interpretation of data: Kyoung Won Kim, Young Seo Cho

Drafting of manuscript: Young Seo Cho, Dagvasumberel Munkhbaatar

Critical revision: Kyoung Won Kim, Bo Hyun Kim, Jeongjin Lee, Gi Won Song, Sung Gyu Lee

\section{Acknowledgements}

This research was supported by the Basic Science Research Program of the National Research Foundation of Korea, funded by the Ministry of Education, Science, and Technology (grant 20100021107).

\section{Conflicts of Interest}

The authors have no conflicts to disclose.

\section{REFERENCES}

1. Jain A, Reyes J, Kashyap R, Dodson SF, Demetris AJ, Ruppert K, et al. Long-term survival after liver transplantation in 4,000 consecutive patients at a single center. Ann Surg 2000;232:490-500.

2. Langnas AN, Marujo W, Stratta RJ, Wood RP, Shaw BW Jr. Vascular complications after orthotopic liver transplantation. Am J Surg 1991;161:76-82; discussion 82-73.
3. Flint EW, Sumkin JH, Zajko AB, Bowen A. Duplex sonography of hepatic artery thrombosis after liver transplantation. AJR Am J Roentgenol 1988;151:481-483.

4. Dodd GD 3rd, Memel DS, Zajko AB, Baron RL, Santaguida LA. Hepatic artery stenosis and thrombosis in transplant recipients: Doppler diagnosis with resistive index and systolic acceleration time. Radiology 1994;192:657-661.

5. Herold C, Reck T, Ott R, Schneider HT, Becker D, Schuppan D, et al. Contrast-enhanced ultrasound improves hepatic vessel visualization after orthotopic liver transplantation. Abdom Imaging 2001;26:597600.

6. Shung KK, Yuan YW, Fei DY, Tarbell JM. Effect of flow disturbance on ultrasonic backscatter from blood. J Acoust Soc Am 1984; 75:1265-1272.

7. Forsberg F, Liu JB, Burns PN, Merton DA, Goldberg BB. Artifacts in ultrasonic contrast agent studies. J Ultrasound Med 1994;13:357365.

8. Yokoyama N, Schwarz KQ, Chen X, Steinmetz SD, Becher H, Schimpky $C$, et al. The effect of echo contrast agent on Doppler velocity measurements. Ultrasound Med Biol 2003;29:765-770.

9. Melany ML, Grant EG, Farooki S, McElroy D, Kimme-Smith C. Effect of US contrast agents on spectral velocities: in vitro evaluation. Radiology 1999;211:427-431.

10. Gutberlet M, Venz S, Zendel W, Hosten N, Felix R. Do ultrasonic contrast agents artificially increase maximum Doppler shift? In vivo study of human common carotid arteries. J Ultrasound Med 1998; 17:97-102.

11. Heiken JP, Brink JA, McClennan BL, Sagel SS, Forman HP, DiCroce J. Dynamic contrast-enhanced CT of the liver: comparison of contrast medium injection rates and uniphasic and biphasic injection protocols. Radiology 1993;187:327-331.

12. Basilico R, Filippone A, Guidotti A, Di Giandomenico V, Muzi M, Bonomo L. Reproducibility of Doppler ultrasonography in the study of hepatic blood flow. Radiol Med 1994;87:268-274.

13. Sacerdoti D, Gaiani S, Buonamico P, Merkel C, Zoli M, Bolondi L, et al. Interobserver and interequipment variability of hepatic, splenic, and renal arterial Doppler resistance indices in normal subjects and patients with cirrhosis. J Hepatol 1997;27:986-992.

14. Hübner GH, Steudel N, Reissmann A, Kühne I, Kleber G, Schärff K, et al. Hepatic arterial doppler sonography in patients with cirrhosis and controls: observer and equipment variability with use of the ultrasonic contrast agent SHU 508A. Z Gastroenterol 2005;43:639645. 